\title{
Magnetohydrodynamic shear instabilities arising from interacting vorticity waves
}

\author{
E. Heifetz \& J. Mak \\ Department of Geophysics \& Planetary Sciences, \\ Tel Aviv University, Israel
}

\begin{abstract}
The presence of a background magnetic field is known to have a stabilising as well as a destabilising effect for shear flow instabilities. To explain the reason for this, we extend the Counter-propagating Rossby Waves mechanism, well known in the geophysical fluid dynamics community, to the magnetohydrodynamic setting. It is demonstrated here that wave displacement leads to a magnetic field configuration that results in an appropriate vorticity distribution, and, via the non-local velocity field generated by the local vorticity anomalies, action-at-a-distance results in a constructive interaction between two waves and leads to shear instability. The existence of shear instability then rests upon whether the chosen basic state supports such a configuration required for constructive interference.

Keywords: shear instability mechanism, magnetohydrodynamics, vorticity actionat-a-distance.
\end{abstract}

\section{Introduction}

Shear flows are ubiquitous in nature, and the process of initial instability, nonlinear development and possible transition to turbulence is relevant to, for example, geophysical and astrophysical systems. In particular, fluids relevant to astrophysical systems (e.g., the solar tachocline, atmospheres of hot exoplanets, the magnetopause) are often ionised, and to describe the interaction of the fluid with the ambient magnetic field requires, at the very least, a magnetohydrodynamic (MHD) description. Of interest here is the physical mechanism leading to MHD shear instabilities. The argument often invoked is that, in the presence of a background magnetic field, the fluid instability has to bend field lines, so one expects the presence of a magnetic field to be stabilising (e.g., [1]). This is 
normally what is observed when the background magnetic field is uniform (e.g., [1-3]), however, it has been reported that a spatially varying magnetic field can cause a destabilisation (e.g., [4-9]). To further add to the confusion, the presence of a uniform magnetic field can destabilise some wavenumbers that are otherwise stable in the hydrodynamic setting (e.g., [5, 6, 10]). Here, a mechanistic interpretation is provided to unify the two observations; this interpretation is related to the negative-energy wave resonance arguments sometimes used to explain shear instabilities (e.g., [11]).

The Counter-propagating Rossby Waves (CRW) framework is well-known in the geophysical fluid dynamics community and dates back to the work of Bretherton [12] and Hoskins et al. [13], as an interpretation for baroclinic and barotropic instability. To illustrate this mechanism, we consider the barotropic case with the Rayleigh profile, with a pictorial schematic is given in figure 1 . The idea is that, by itself, each Rossby wave supported on the background vorticity gradient is neutral. For the top wave, we have the relation $q \sim-\Delta \bar{Q} \eta$, where $q$ is the vorticity, $\Delta \bar{Q}$ is the jump in the background vorticity (positive at the location of the top wave), and $\eta$ is the cross-stream displacement. Displacement of the material surface results in a vorticity distribution that leads to the wave propagating against the background flow, in this case to the left. Taking into account that $\Delta \bar{Q}<0$ for the bottom wave, we see that the bottom wave propagates to the right, but counter to the background flow. The counter-propagation results in scenarios where the waves can be held stationary against the background mean flow, resulting in phaselocking. Further, in the presence of the other wave, the local vorticity anomalies induce a non-local velocity field (that decays away from the source), resulting in interaction between the two waves. This interaction helps the other maintain phaselocking [13], and, with an appropriate phase difference, constructive interference can result, where each wave amplifies the other's displacement. This serves as an explanation for modal instability in barotropic and baroclinic instability. Details such as transient growth/non-modal instabilities, non-localised generation of vorticity, initial value evolution of waves not in the configuration of figure 1 have also been investigated, and we refer the reader to the recent review of [14] and references within for more details.

The scenario demonstrated in figure 1 uses the Rayleigh profile in the barotropic setting as an illustrative example, where the waves with associated vorticity anomalies arise from the fact that there is a gradient in the background vorticity profile. This is of course not the only possible scenario, and it has been demonstrated that a similar wave interpretation may be applied to stratified shear flow instabilities. Displacement of the individual waves (gravity, Rossby or mixed) induces a buoyancy distribution that results in an appropriate vorticity distribution depending on the type of waves that are supported (e.g., $[15,16])$. The resulting wave interaction differs in the details, but the important point is that a schematic similar to figure 1 holds also in the stratified setting, whether it is instability of the Kelvin-Helmholtz type (two vorticity waves, [15]), Holmboe type (one vorticity and one gravity wave, $[15,17]$ ) or the Taylor-Caulfield type (two gravity waves, $[18,19])$. In particular, the background flow can be a linear shear flow, i.e., 

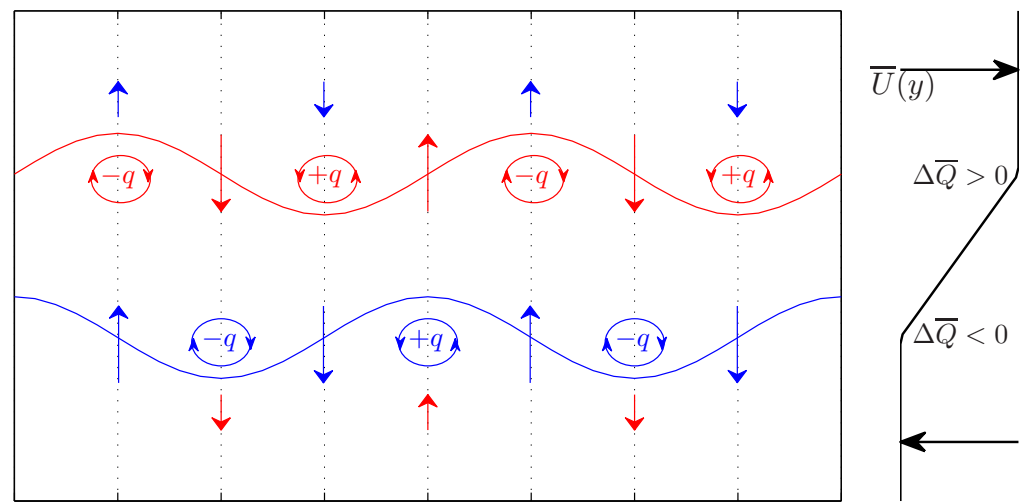

Figure 1: A pictorial representation of the CRW mechanism. Mutual interaction is via the non-local velocity field generated by the local vorticity anomalies, resulting in phase-locking and mutual amplification. The configuration presented here is with the optimum configuration for growth, with an absolute phase difference of $\pi / 2$ and each wave with equal amplitude.

no background vorticity gradient, and instability of the Taylor-Caufield type can still arise [20,21]; see [22], [14], and references within for details.

We demonstrate here that a similar process to the stratified case occurs in the MHD system. Displacement leads to a magnetic field configuration that results in a vorticity distribution associated with waves that may be of Rossby, Alfvén, or mixed type. The stabilisation or destabilisation of the presence of the background magnetic field then rests on the ability whether waves can phase-lock and achieve mutual amplification. For space considerations, we focus here only on rationalising the physics associated with the wave dynamics; the details of all the associated analysis and concrete calculations with canonical basic states will be presented elsewhere.

\section{Generation of vorticity by the Lorentz force}

The two-dimensional, incompressible, homogeneous MHD equations are given by

$$
\begin{aligned}
\frac{\partial \boldsymbol{u}}{\partial t}+\boldsymbol{u} \cdot \nabla \boldsymbol{u} & =-\frac{1}{\rho_{0}} \nabla p+\frac{1}{\mu_{0} \rho_{0}} j^{*} \times \boldsymbol{B}^{*}, \\
\frac{\partial \boldsymbol{B}^{*}}{\partial t}+\boldsymbol{u} \cdot \nabla \boldsymbol{B}^{*} & =\boldsymbol{B}^{*} \cdot \nabla \boldsymbol{u},
\end{aligned}
$$

with $\nabla \cdot \boldsymbol{u}=0$ and $\nabla \cdot \boldsymbol{B}^{*}=0 ; \boldsymbol{j}^{*}=\nabla \times \boldsymbol{B}^{*}$ is the current. Since we assume incompressibility, the velocity field and the magnetic field $\boldsymbol{B}=\boldsymbol{B}^{*} / \sqrt{\mu_{0} \rho_{0}}$ 
(where $\mu_{0}$ is the permeability of free-space and $\rho_{0}$ is the constant density, so that $\boldsymbol{B}$ is in units of velocity) may be written in terms of a (scalar) streamfunction $\psi$ and magnetic potential $A$, defined here as

$$
\boldsymbol{u}=\boldsymbol{e}_{z} \times \nabla \psi, \quad \boldsymbol{B}=\boldsymbol{e}_{z} \times \nabla A
$$

Then we observe that the vorticity $q$ and current $j$ satisfies the relations $q=\nabla^{2} \psi$ and $j=\nabla^{2} A$. The full equations now takes the equivalent form

$$
\frac{D q}{D t}=\nabla \cdot(j \boldsymbol{B}), \quad \frac{D A}{D t}=0,
$$

where $D / D t$ is the material derivative. Vorticity is now no longer materially conserved as the Lorentz force, written in terms of a flux divergence, can generate vorticity accordingly.

Just from looking at the form of the forcing in the vorticity equation above, we expect immediately that both $\partial\left(j b_{x}\right) / \partial x>0$ and $\partial\left(j b_{y}\right) / \partial y>0$ leads to positive vorticity generation. To illustrate this, suppose we have a constant $B_{0} \boldsymbol{e}_{x}$ with $B_{0}>0$, and

$$
j\left(x_{0}, y_{0}\right) \boldsymbol{e}_{z}=j_{0} \boldsymbol{e}_{z}, \quad j\left(x_{1}, y_{1}\right) \boldsymbol{e}_{z}=j_{1} \boldsymbol{e}_{z}
$$

where $j_{1}>j_{0}, x_{1}>x_{0}, y_{1}=y_{0}$. Now, in general, the Lorentz term is $\boldsymbol{F}=\boldsymbol{j} \times \boldsymbol{B}$, and so, taking $\boldsymbol{e}_{z}$ to point out of the page, the current distribution above produces an acceleration

$$
\boldsymbol{F}\left(x_{0}, y_{0}\right)=F_{0} \boldsymbol{e}_{y}, \quad \boldsymbol{F}\left(x_{1}, y_{1}\right)=F_{1} \boldsymbol{e}_{y}
$$

with $F_{1}>F_{0}>0$. Since this is an acceleration increasing in $x$, a material line connecting $\left(x_{0}, y_{0}\right)$ and $\left(x_{1}, y_{1}\right)$ is rotated anti-clockwise, i.e., we have positive vorticity production associated with a $\partial\left(j b_{x}\right) / \partial x>0$ configuration. Similarly, to construct a $\partial\left(j b_{y}\right) / \partial y>0$ scenario, we take $B_{0} \boldsymbol{e}_{y}$, the above current distribution but taking $x_{1}=x_{0}$ and $y_{1}>y_{0}$ (together with the analogous assumptions), this yields

$$
\boldsymbol{F}\left(x_{0}, y_{0}\right)=-F_{0} \boldsymbol{e}_{y}, \quad \boldsymbol{F}\left(x_{1}, y_{1}\right)=-F_{1} \boldsymbol{e}_{y},
$$

where $F_{1}>F_{0}$, and this may be seen to produce positive vorticity. A pictorial representation of this is given in figure 2 .

\section{Interfacial wave dynamics}

To demonstrate how vorticity anomalies arise from the interfacial wave modes supported in the MHD system, we linearise the full equations about a basic state $\bar{U}(y) \boldsymbol{e}_{x}$ and $\bar{B}(y) \boldsymbol{e}_{x}$ (and thus $\bar{Q}=-\partial \bar{U} / \partial y$ and $\bar{J}=-\partial \bar{B} / \partial y$ ). The governing 

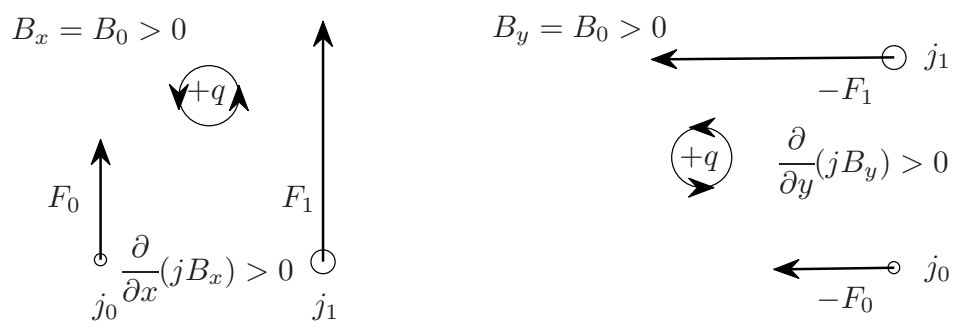

Figure 2: Generation of vorticity by the Lorentz force resulting from a magnetic field configuration; see text for details.

equations are then

$$
\begin{aligned}
\left(\frac{\partial}{\partial t}+\bar{U} \frac{\partial}{\partial x}\right) q & =-\frac{\partial \bar{Q}}{\partial y} \frac{\partial \psi}{\partial x}+\bar{B} \frac{\partial j}{\partial x}+\frac{\partial \bar{J}}{\partial y} \frac{\partial A}{\partial x}, \\
\left(\frac{\partial}{\partial t}+\bar{U} \frac{\partial}{\partial x}\right) A & =\bar{B} \frac{\partial \psi}{\partial x} .
\end{aligned}
$$

Substituting for the cross-stream displacement $\eta$ in equation (7b), where

$$
v=\frac{\partial \psi}{\partial x}=\left(\frac{\partial}{\partial t}+\bar{U} \frac{\partial}{\partial x}\right) \eta
$$

an integration gives the relation $A=\bar{B} \eta$ (plus a possible non-advective contribution $\tilde{A}(y)$ that is assumed to be zero here).

For the case where the domain is periodic in $x$ and unbounded in $y$, the relations $q=\nabla^{2} \psi$ and $j=\nabla^{2} A$ may be formally inverted via a Green's function; in this case, the explicit relation is given by

$$
\left(\begin{array}{l}
A(y) \\
\psi(y)
\end{array}\right)=-\frac{1}{2 k} \int\left(\begin{array}{l}
j\left(y^{\prime}\right) \\
q\left(y^{\prime}\right)
\end{array}\right) \mathrm{e}^{-k\left|y-y^{\prime}\right|} \mathrm{d} y^{\prime}
$$

With this, we suppose that $\bar{U}$ and $\bar{B}$ are piecewise-continuous at $y=L$, and

$$
\frac{\partial \bar{Q}}{\partial y}=\Delta \bar{Q} \delta(y-L), \quad \frac{\partial \bar{J}}{\partial y}=\Delta \bar{J} \delta(y-L)
$$

Then, assuming interfacial wave solutions of the form

$$
q=\hat{q} \mathrm{e}^{-\mathrm{i} k c t} \delta(y-L), \quad j=\hat{j} \mathrm{e}^{-\mathrm{i} k c t} \delta(y-L),
$$


and observing that $(\psi(L), A(L))=(-\hat{q} / 2 k,-\hat{j} / 2 k)$ via the inversion, the governing set of equations then becomes, upon using the relation $A=\bar{B} \eta$,

$$
\begin{aligned}
& (\bar{U}-c) \hat{q}=\frac{\Delta \bar{Q}}{2 k} \hat{q}-2 k \bar{B}\left(\bar{B}-\frac{\Delta \bar{J}}{2 k}\right) \eta \\
& (\bar{U}-c) \eta=-\frac{1}{2 k} \hat{q},
\end{aligned}
$$

where $\bar{U}$ and $\bar{B}$ are taken to be evaluated at $y=L$. The eigenstructure then reads $\hat{q}^{ \pm}=2 k\left(c^{ \pm}-\bar{U}\right) \eta^{ \pm}$, with the dispersion relation

$$
\left(c^{ \pm}-\bar{U}\right)=-\frac{\Delta \bar{Q}}{4 k} \pm \sqrt{\left(\frac{\Delta \bar{Q}}{4 k}\right)^{2}+\bar{B}\left(\bar{B}-\frac{\Delta \bar{J}}{2 k}\right)} .
$$

We will call these waves generalised interfacial Rossby-Alfvén waves. We denote the branches as the 'plus' and 'minus' branch depending on whether the plus or minus sign is taken in (13); we note that the minus branch is associated with a wave propagating counter to the background flow, and vice versa for the plus branch.

The $\Delta \bar{Q}, \bar{B}^{2}$ and $\bar{B} \Delta \bar{J}$ terms in (13) are associated with the first, second and third terms in $(7 \mathrm{a})$. To rationalise how these terms are associated with vorticity anomalies, we consider how the individual components generate vorticity that can lead to wave propagation, or, as the reader may have noticed, potentially to an instability of the interface.

With only the $\Delta \bar{Q}$ terms, we recover the Rossby modes, and propagation of the wave from its associated vorticity anomalies are as in figure 1. For the terms that arise from MHD effects, we first suppose we only have the contribution from the $\bar{B}^{2}$ term in the absence of the other terms, i.e., vorticity is generated by the $\bar{B}(\partial j / \partial x)$ term in (7a). We first note that we recover (shear) Alfvén wave modes, which we will call 'pure' Alfvén waves to distinguish these with Alfvén waves that are supported when there is a background current gradient. Assuming for simplicity $\bar{B}>0$, the relation $A=\bar{B} \eta$ results in $\eta \sim-j$ since the Laplacian operator is negative-definite. With the wave displacement as in the top panel of figure 3 , the resulting $j$ distribution along with $b_{x}=\bar{B}$ results in a vorticity distribution centred at the nodes accordingly. The induced velocity acts to reduce the wave amplitude, and to maintain the wave structure, the vorticity distribution required should be in phase or anti-phase with the displacement, as in the bottom two panels respectively. When the two are in phase, i.e., $\eta \sim q$, we have a wave propagating to the right, and when the two are in anti-phase, i.e., $\eta \sim-q$, we have a wave propagating to the left, relative to the background flow; this is consistent with the eigenstructure of the plus and minus branch, with the mathematical form given in (13).

For the $\bar{B}(\partial A / \partial x)=\bar{B} \Delta \bar{J}(\partial \eta / \partial x)$ term in (7a), we neglect the $\bar{B}(\partial j / \partial x)$ contribution for the moment, and note that we need to add this back on afterwards since both are present when $\bar{B}>0$. As before, we have $\eta \sim-j$, and we can have two scenarios depending on the sign of $\Delta \bar{J}$. When $\Delta \bar{J}<0$ so that $\bar{B} \Delta \bar{J}<0$, 

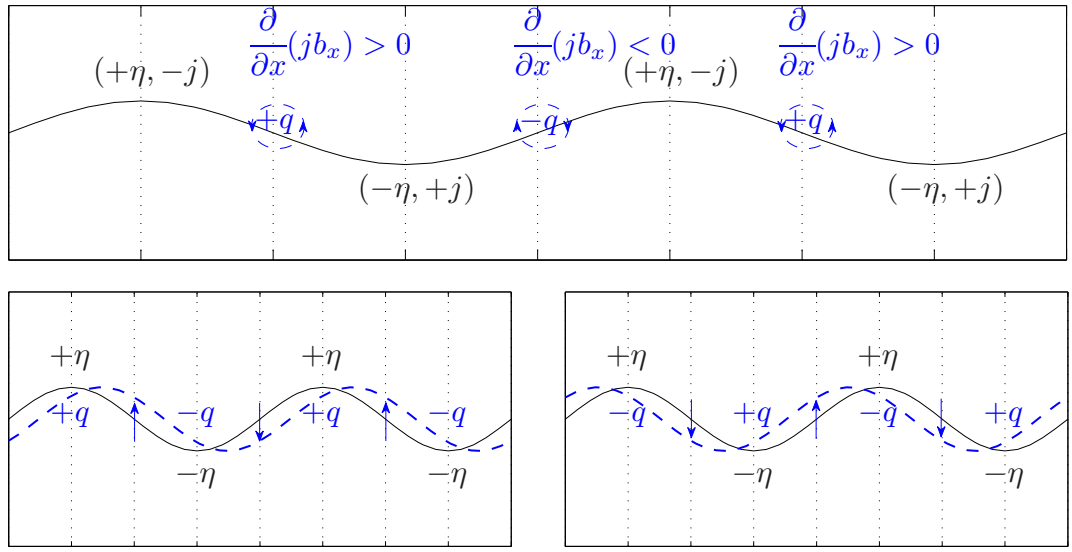

Figure 3: Wave propagation associated with the $\bar{B}(\partial j / \partial x)$ term in (7a); see text for details.

the scenario is as in the top panel of figure 4. The resulting current distribution generates a $b_{y}$ distribution denoted by the black arrows, and such a configuration generates a vorticity distribution at the nodes accordingly. Then we notice that this is exactly like the previous case of the pure Alfvén waves in figure 3, and the cumulative effect of the two contributions results in a faster wave propagation. All of the schematics may be seen to be consistent with the mathematical form of the eigenstructure and dispersion relation in (13), taking $\bar{B} \Delta \bar{J}<0$.

With $\Delta \bar{J}$ and $\bar{B} \Delta \bar{J}>0$, the situation is as depicted in the bottom panel of figure 4 , and the resulting vorticity distribution then acts to enhance the displacement of the wave. There is competition then between the propagation part from $\bar{B}(\partial j / \partial x)$ and the destabilising part from $\bar{B} \Delta \bar{J}(\partial \eta / \partial x)$. From the eigenstructure, we see that the destabilising component always dominates in the small $k$ regime. We have a steepening of the wave whether $q$ is in phase or anti-phase with the displacement, and we therefore conclude that, in the small $k$ regime, the interface itself may be unstable even without a background flow, in a standing wave fashion similar to the Rayleigh-Taylor instability. Since the interest is really in shear instabilities, we do not discuss this further here, and we refer the interested reader to the discussion in the longer version of the article to be presented elsewhere.

\section{Conclusion}

Having derived and rationalised how displacement of MHD wave modes has associated with it vorticity anomalies, via the change in the magnetic field configuration, the presence of two or more of these neutral waves can then lead to shear instability via vorticity inversion, action-at-a-distance and mutual 

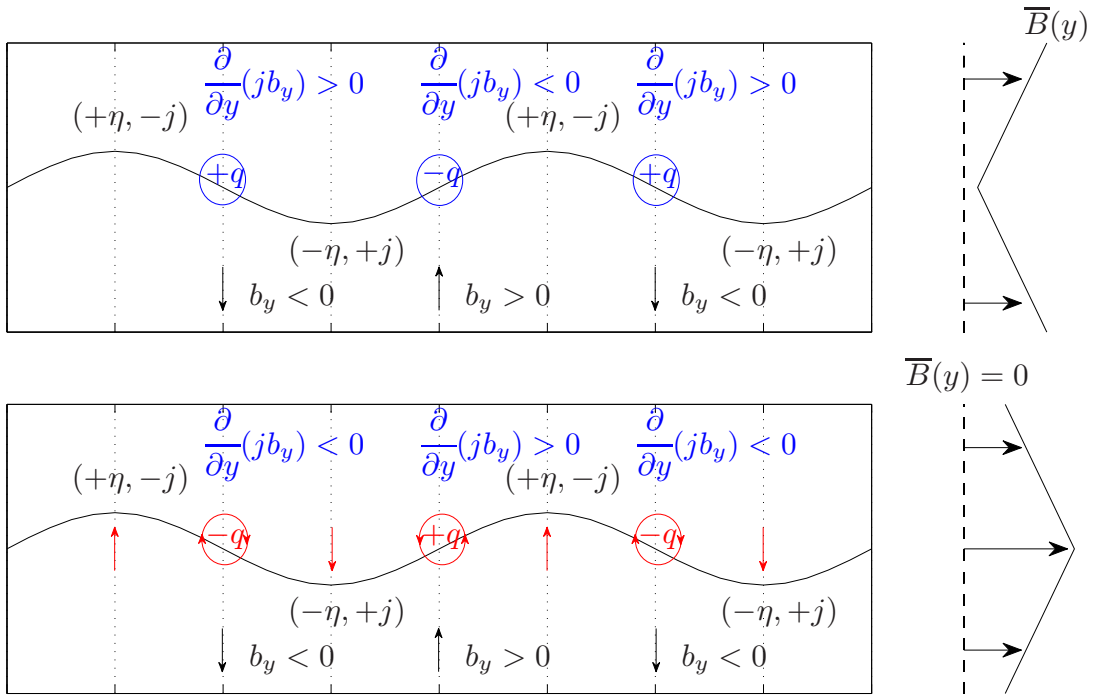

Figure 4: Wave propagation and potential instability associated with the $\bar{B}(\partial A / \partial x)=\bar{B} \Delta \bar{J}(\partial \eta / \partial x)$ term in (7a); see text for details.

interaction. The exact details of the shear instability depends on the individual waves types supported by the choice of the basic state, but the main point is that these fine details are minor additions to the fundamental configuration depicted in figure 1. At the simplest level, whether the presence of the magnetic field is stabilising or destabilising boils down to how the set up changes phase-locking and associated interaction of the waves.

We have focussed here on a physical explanation and rationalisation of the relation between displacement and vorticity generation, and due to space considerations we have omitted some finer details. Further analysis have been carried out for two specific profiles that illustrates, (i) a case where the magnetic field stabilises a flow that is hydrodynamically unstable, and (ii) a case where the magnetic field destabilises a flow that is hydrodynamically stable. The role of the pro- and counter-propagating components, the critical layer, and a comparison of the closed form analytical solutions with the full numerical results have been carried out; the details of these will be presented elsewhere.

A portion of this work was carried out whilst both authors were visiting the Department of Meteorology, Stockholm University. The authorship is alphabetical.

\section{References}

[1] Chandrasekhar, S., Hydrodynamic and hydromagnetic stability. Dover Publications Inc., dover edition, 1981. 
[2] Hughes, D.W. \& Tobias, S.M., On the instability of magnetohydrodynamic shear flows. Proc R Soc Lond A, 457, pp. 1365-1384, 2001.

[3] Mak, J., Shear instabilities in shallow-water magnetohydrodynamics. Ph.D. thesis, University of Leeds, 2013.

[4] Stern, M.E., Joint instability of hydromagnetic fields which are separately stable. Phys Fluids, 6, pp. 636-642, 1963.

[5] Kent, A., Instability of laminar flow of a perfect magnetofluid. Phys Fluids, 9, p. 1286, 1966.

[6] Kent, A., Stability of laminar magnetofluid flow along a parallel magnetic field. J Plasma Phys, 2, pp. 543-556, 1968.

[7] Chen, X.L. \& Morrison, P.J., A sufficient condition for the ideal instability of shear flow with parallel magnetic field. Phys Fluids B, 3, p. 863-865, 1991.

[8] Tatsuno, T. \& Dorland, W., Magneto-flow instability in symmetric field profiles. Phys Plasmas, 13, p. 092107, 2006.

[9] Lecoanet, D., Zweibel, E.G., Townsend, R.H.D. \& Huang, Y.M., Violation of Richardson's criterion via introduction of a magnetic field. Astrophys J, 712, pp. 1116-1128, 2010.

[10] Ray, T.P. \& Ershkovich, A.I., Kelvin-Helmholtz instabilities in a sheared compressible plasma. Mon Not R Astron Soc, 204, pp. 821-831, 1983.

[11] Cairns, R.A., The role of negative energy waves in some instabilities of parallel flows. J Fluid Mech, 92, pp. 1-14, 1979.

[12] Bretherton, F.P., Baroclinic instability and the short wavelength cut-off in terms of potential vorticity. Q J Roy Met Soc, 92, pp. 335-345, 1966.

[13] Hoskins, B.J., McIntyre, M.E. \& Robertson, A.W., On the use and significance of isentropic potential vorticity maps. $Q J$ Roy Met Soc, 111, pp. 877-946, 1985.

[14] Carpenter, J.R., Tedford, E.W., Heifetz, E. \& Lawrence, G.A., Instability in stratified shear flow: Review of a physical interpretation based on interacting waves. Appl Mech Rev, 64, p. 061001, 2012.

[15] Baines, P.G. \& Mitsudera, H., On the mechanism of shear instabilities. J Fluid Mech, 276, pp. 327-342, 1994.

[16] Harnik, N., Heifetz, E., Umurhan, O.M. \& Lott, F., A buoyancy-vorticity wave interaction approach to stratified shear flow. J Atmos Sci, 65, pp. 26152630, 2008.

[17] Holmboe, J., On the behaviour of symmetric waves in stratified shear layers. Geophys Publ, 24, pp. 67-113, 1962.

[18] Taylor, G.I., Effect of variation in density on the stability of superposed streams of fluid. Proc R Soc Lond A, 132, pp. 499-523, 1931.

[19] Caulfield, C.P., Multiple linear instability of layered stratified shear flow. J Fluid Mech, 258, p. 255-285, 1994.

[20] Rabinovich, A., Umurhan, O.M., Harnik, N., Lott, F. \& Heifetz, E., Vorticity inversion and action-at-a-distance instability in stably stratified shear flow. J Fluid Mech, 670, pp. 301-325, 2011. 
380 Advances in Fluid Mechanics X

[21] Guha, A. \& Lawrence, G.A., A wave interaction approach to studying nonmodal homogeneous and stratified shear instabilities. submitted to J Fluid Mech, 2013.

[22] Carpenter, J.R., Balmforth, N.J. \& Lawrence, G.A., Identifying unstable modes in stratified shear layers. Phys Fluids, 22, p. 054104, 2012. 